\title{
Numerical Study of Creeping Flow Through Sinusoidally Periodic Tube
}

\author{
Asif Mahmud ${ }^{1}$, Suhana Perveen ${ }^{1}$, Md. Nazmul Hasan ${ }^{1}$, Md. Samsuzzoha ${ }^{2}$, Nazmul Islam ${ }^{1,}$ * \\ ${ }^{1}$ Mathematics Discipline, Khulna University, Khulna, Bangladesh \\ ${ }^{2}$ Department of Mathematics, Swinburne University of Technology, Victoria, Australia
}

Email address:

nazmul.islam@math.ku.ac.bd (N. Islam)

${ }^{*}$ Corresponding author

\section{To cite this article:}

Asif Mahmud, Suhana Perveen, Md. Nazmul Hasan, Md. Samsuzzoha, Nazmul Islam. Numerical Study of Creeping Flow Through Sinusoidally Periodic Tube. International Journal of Applied Mathematics and Theoretical Physics. Vol. 5, No. 3, 2019, pp. 72-81. doi: 10.11648/j.ijamtp.20190503.14

Received: April 29, 2019; Accepted: September 4, 2019; Published: September 19, 2019

\begin{abstract}
There has been renewed interest in the flow behaviour within tubes with periodically varying cross-section with the recognition that they can be used as particle separation devices. In this paper, we present a numerical study of the effect of tube geometry on creeping flow of viscous incompressible fluid through sinusoidally constricted periodic tube which is axisymmetric but longitudinally asymmetric. The boundary element method is used to solve for the flow in the tube by specifying the pressure drop across the ends of the tube. The boundary element equations have been formulated for an infinite periodic tube by writing the velocity in terms of the integrals over the tube boundary and is used to calculate the force on the tube boundary, to obtain the detailed velocity distribution within the tube and to determine the effect of amplitude and wavelength of corrugation on the structure of the flow. We have found that the highest axial velocity is at throat region and lowest axial velocity is at expansion region. Also, we have discovered that the maximum radial velocity occurs at diverging cross-section and minimum radial velocity occurs at converging cross-section. The tangential force on the tube wall is examined for different amplitudes and wavelengths of corrugation and observed that the tangential force is greater in the constricted region than in the expansion region. The physical quantities (such as velocity and force) increase with increasing amplitude and decrease with increasing wavelength. Finally, we have compared our results with the work of Hemmat and Borhan [3] and have found good agreement with them.
\end{abstract}

Keywords: Creeping Flow, Numerical Study, Periodic Tube, Sinusoidal Cross-Section, Boundary Element Method

\section{Introduction}

Fluid dynamics comprehends excellent opportunity to study the flow of fluids through axisymmetric and periodically constricted periodic tube and this has been an engrossing topic in recent times [1-6]. This type of research has been used widely in different sectors such as separation of colloidal and sub-colloidal particles through micro-fluid and nano-fluidic devices [7]. It can also be used in modeling transport process in porous media [8]. Nowadays researchers are using this flow system to examine the analysis of flow in shriveled velum, simulated lung and arterial prostheses etc. [3].

In early 1800's Hagen [9] and Pouseuille [10] examined the Newtonian flow through axisymmetric periodic tube. Since then, many scientists expanded their attention to subsume nonNewtonian flows [11] in an open channel [12], tubes with corner [13] and tube with restrictions [11] etc. All of the scientist overrates mostly a flow through axisymmetric tube but they have taken their consideration in the fact that the radius of this tube varies periodically with respect to its length [1-6].

There have been many experimental and theoretical studies of flow through periodically constricted capillaries. On the experimental side, Forrester and Young [14], Deibar and Schowalter [2], Leneweit and Auerbach [15], studied the fluid flow through periodic tube with different cross section for a wide range of Reynolds number, i.e., for higher as well as for lower Reynolds number and visualized the flow structure for low and high Reynolds 
number. In contrast, Nishimura et al. [16] experimentally observed the flow structure within sinusoidally constricted periodic tube only for higher Reynolds number.

On the theoretical side Chow \& Soda [1] and Sisavath et al. [8] studied the flow through sinusoidally periodic tube and obtained asymptotic series solution of the Navier-Stokes equation at moderate Reynolds number. They have found good agreement with the result of Deiber and Schowalter [2]. A number of numerical techniques, such as spectral methods [3], iterative method [4], and finite difference technique [5] have also been used to solve the full Navier-Stokes equation.

The creeping flow through sinusoidally constricted periodic capillary has been studied by Hemmat and Borhan and solved governing equation (Stoke's and continuity equation) by using boundary element method to find the detailed velocity distribution within one-wave section. Recently, Islam et al. [17] studied the creeping flow through different periodic tube such as co-sinusoidal, parabolic, triangular and saw-tooth tube profiles. They formulated the BEM over an infinite periodic tube with pressure condition and obtained velocity distribution in the tube profiles. However, they didn't study the detailed velocity distribution for a sinusoidal tube profile which is considered to be axisymmetric but longitudinally asymmetric.

Hence in this paper, we have investigated the creeping flow through sinusoidally constricted periodic tube profile which is axisymmetric but longitudinally asymmetric. We have studied low Reynolds number flow in order to examine the effect of geometric parameters, such as amplitude, wavelength of corrugation on the flow structure. We have formulated boundary element method for infinite number of wave-sections with pressure condition.

\section{Mathematical Formulation}

Consider the creeping flow of an incompressible Newtonian fluid through an infinite axisymmetric periodic tube. An infinite sinusoidally constricted capillary is considered so that we have used pressure difference boundary condition. The flow is governed by a pressure gradient $\Delta P / L$, where $\Delta P$ is the dimensional pressure drop across the wavelength of the tube and $L$ is the wavelength of one wave-section of the tube.

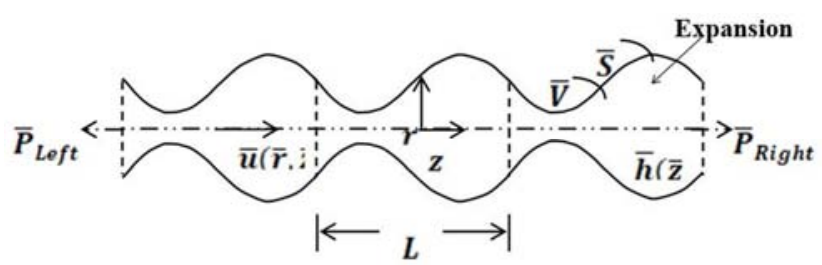

Figure 1. Schematic view of an infinitely long, cylindrical periodically constricted capillary $\bar{h}(\bar{z})=\bar{h}(\bar{z}+k L)$.

The surface of the tube is $\boldsymbol{y}=\overline{\boldsymbol{z}} \hat{\mathbf{z}}+\bar{h}(\bar{z}) \hat{\mathbf{r}}$, where $\hat{\mathbf{r}}$ and $\hat{\mathbf{z}}$ are unit vectors in the radial and longitudinal directions, respectively, $\bar{h}(\bar{z})$ defines the tube profile. The governing equations in the absence of inertial effects (small Reynolds number, Stokes Flow) can be written in dimensional variables as

$$
\bar{\nabla}^{2} \overline{\boldsymbol{u}}=\frac{1}{\mu} \bar{\nabla} p, \bar{\nabla} \cdot \overline{\boldsymbol{u}}=0
$$

where $\overline{\boldsymbol{u}}(\bar{r}, \bar{z})$ is the fluid velocity, $\bar{p}=(\bar{r}, \bar{z})$ is the fluid pressure and $\mu$ is the viscosity of the fluid.

For an axisymmetric problem, we have

$$
\bar{\nabla}=\frac{\partial}{\partial \bar{r}} \hat{\mathbf{r}}+\frac{\partial}{\partial \bar{z}} \hat{\mathbf{z}}
$$

We have considered no slip boundary conditions on the tube surface $\bar{S}$, so that $\overline{\boldsymbol{u}}(\overline{\boldsymbol{x}})=0$ for $\overline{\boldsymbol{x}} \in \bar{S}$.

On introducing the non-dimensional variables,

$$
z=\frac{\bar{z}}{L}, r=\frac{\bar{r}}{L}, \quad p=\frac{\bar{p}}{P_{0}}, \quad \boldsymbol{u}=\frac{\overline{\boldsymbol{u}} \mu}{\left(L P_{0}\right)}
$$

the governing equations for the viscous flow can be written in non-dimensional form as

$$
\nabla^{2} \boldsymbol{u}=\nabla p, \nabla \cdot \boldsymbol{u}=0
$$

while the no slip boundary conditions become,

$$
u(x)=0 \text { for } x \in S
$$

The fundamental solutions of Stokes equation are [18]

$$
\begin{aligned}
& \boldsymbol{u}(\boldsymbol{x})=\frac{1}{4 \pi} \int_{S} d S(\boldsymbol{y}) \mathbf{G}(\boldsymbol{x}, \boldsymbol{y}) \cdot \mathbf{F}(\boldsymbol{y})-\frac{1}{4 \pi} \int_{S} d S(\boldsymbol{y}) \mathbf{H}(\boldsymbol{x}, \boldsymbol{y}) \cdot \boldsymbol{u}(\boldsymbol{y}) \\
& \boldsymbol{u}(\boldsymbol{x})=\frac{1}{8 \pi} \int_{S} d S(\boldsymbol{y}) \mathbf{G}(\boldsymbol{x}, \boldsymbol{y}) \cdot \mathbf{F}(\boldsymbol{y})-\frac{1}{4 \pi} \int_{S} d S(\boldsymbol{y}) \mathbf{H}(\boldsymbol{x}, \boldsymbol{y}) \cdot \boldsymbol{u}(\boldsymbol{y})
\end{aligned}
$$

where, $d S$ is the surface area element of the boundary $S$ at $\boldsymbol{y}$ and $V$ is the interior region of the tube. In addition, $\mathbf{F}(\boldsymbol{y})=\sum(\boldsymbol{y}) \cdot \hat{\boldsymbol{n}}(\boldsymbol{y})$ is the force per unit area exerted on the fluid by the boundary at $y$ (boundary force), where $\sum(\boldsymbol{y})$ is the stress tensor which is defined as

$$
\sum_{i j}=-p \delta_{i j}+\left(\frac{\partial u_{i}}{\partial x_{j}}+\frac{\partial u_{j}}{\partial x_{i}}\right)
$$


and the normal vector, $\hat{\boldsymbol{n}}(\boldsymbol{y})=\left(n_{r}, n_{z}\right)$ is directed outward from the control volume, $V$. Also $\boldsymbol{G}(\boldsymbol{x}, \boldsymbol{y})$ and $\boldsymbol{H}(\boldsymbol{x}, \boldsymbol{y})$ are functions of the sample point $\boldsymbol{x}$ and source point $\boldsymbol{y}$ which are defined as follows

$$
G_{i j}(\boldsymbol{x}, \boldsymbol{y})=\frac{\delta_{i j}}{\epsilon}+\frac{\hat{x}_{i} \hat{x}_{j}}{\epsilon^{3}} \text { and } H_{i j}(\boldsymbol{x}, \boldsymbol{y})=-6 \frac{\hat{x}_{i} \hat{x}_{j} \hat{x}_{k}}{\epsilon^{5}} n_{k}
$$

where, $\in=|\hat{\boldsymbol{x}}|$ and $\hat{\boldsymbol{x}}=\boldsymbol{y}-\boldsymbol{x}$.

The tangential force on the tube is defined as

$$
F_{t}=F_{z} n_{r}-F_{r} n_{z}
$$

where, $F_{z}$ and $F_{r}$ are the $z$-and $r$-components of $\mathbf{F}(\boldsymbol{x})$, the force per unit area exerted on the fluid by the wall.

\section{Numerical Computation}

We have used Boundary Element Method (BEM) to solve for the flow in the tube. The advantage of this technique is that discretisation need only be performed over the boundary (not over the whole domain) since the velocity field can be written in terms of integrals over the boundary (as in Eqs. (1)-(2)). If the total flux through the tube has been specified, then the velocity profile across the ends of one wave section can be calculated. Since the velocity on the boundary of one wave section is then everywhere known, the boundary element equations (1) can be solved in one representative wave section to calculate the force on the tube surface, $\mathbf{F}(x)$. Once this force is known, the tangential force on the tube surface, $F_{t}$ can be calculated and the fluid velocity can be calculated anywhere within the tube using (2). Since the tube is periodic, this one wave section gives a representation of the flow in each section of the tube. Indeed, this technique has been used in previous studies [3].

In a laboratory situation, it is more common to specify the pressure drop across the ends of the tube, rather than the flux. In this case, the boundary element equations cannot be solved for just one representative wave section as the velocity profile across the ends of one wave section cannot be calculated so that the velocity on the boundary of one wave section (the left of Eq. (1)) is not known everywhere. For this reason, we consider an infinitely long tube, see Figure 1 (b), and formulate the boundary element equations over the entire length of the tube. In this way, the velocity profile at the end cross-sections of the tube does not influence the calculation.

We have written the boundary force as a periodic component by introducing

$$
\mathbf{F}\left(y^{\prime}\right)=z^{\prime} \hat{n}\left(y^{\prime}\right)+\mathbf{f}\left(y^{\prime}\right)
$$

where

$$
f\left(\boldsymbol{y}^{\prime}\right)=\mathbf{f}\left(h\left(z^{\prime}\right) \hat{\mathbf{r}}, z^{\prime} \hat{\mathbf{z}}\right)=\mathbf{f}(h(z) \hat{\mathbf{r}},(z+k) \hat{\mathbf{z}})
$$

is periodic over the length of the tube $(k \in Z)$. Here the fluid velocity on the surface of tube is zero, $\mathbf{u}(\mathbf{x})=\mathbf{0}$ for $\boldsymbol{x} \in S$ and for the long periodic tube, the term $G_{i, j}$ in equation (3) approach zero. Here, we consider axisymmetric tube shapes. That's why we have to reduce the surface integrals in Equation (1) and (2) into line integrals by performing the azimuthal integration analytically in a cylindrical coordinate system. For an infinite periodic tube, equation (1) can be written as

$$
\begin{aligned}
\boldsymbol{u}(\boldsymbol{x})= & \frac{1}{4 \pi} \int_{l(-N-1 / 2)}^{l(N+1 / 2)} d l\left(\boldsymbol{y}^{\prime}\right) \boldsymbol{M}\left(\boldsymbol{x}, \boldsymbol{y}^{\prime}\right) \cdot \mathbf{F}\left(\boldsymbol{y}^{\prime}\right)+\frac{1}{4 \pi} \int_{0}^{h(1 / 2)} d r \boldsymbol{M}\left(\boldsymbol{x},\left(N+\frac{1}{2}\right) \hat{\mathbf{z}}+r \hat{\mathbf{r}}\right) \cdot \mathbf{F}\left(\left(N+\frac{1}{2}\right) \hat{\mathbf{z}}+r \hat{\mathbf{r}}\right) \\
& +\frac{1}{4 \pi} \int_{0}^{h(-1 / 2)} d r \boldsymbol{M}\left(\boldsymbol{x},-\left(N+\frac{1}{2}\right) \hat{\mathbf{z}}+r \hat{\mathbf{r}}\right) \cdot \mathbf{F}\left(-\left(K+\frac{1}{2}\right) \hat{\mathbf{z}}+r \hat{\mathbf{r}}\right)-\frac{1}{4 \pi} \int_{l(-N-1 / 2)}^{l(N+1 / 2)} d l\left(\boldsymbol{y}^{\prime}\right) \boldsymbol{Q}\left(\boldsymbol{x}, \boldsymbol{y}^{\prime}\right) \cdot \boldsymbol{u}\left(\boldsymbol{y}^{\prime}\right) \\
& -\frac{1}{4 \pi} \int_{0}^{h(1 / 2)} d r \boldsymbol{Q}\left(\boldsymbol{x},-\left(N+\frac{1}{2}\right) \hat{\mathbf{z}}+r \hat{\mathbf{r}}\right) \cdot \mathbf{u}\left(-\left(N+\frac{1}{2}\right) \hat{\mathbf{z}}+r \hat{\mathbf{r}}\right)-\frac{1}{4 \pi} \int_{0}^{h(1 / 2)} d r \boldsymbol{Q}\left(\boldsymbol{x},\left(N+\frac{1}{2}\right) \hat{\mathbf{z}}+r \hat{\mathbf{r}}\right) \cdot \boldsymbol{u}\left(\left(N+\frac{1}{2}\right) \hat{\mathbf{z}}+r \hat{\mathbf{r}}\right)
\end{aligned}
$$

where $M$ and $Q$ are related to $G$ and $H$ through an angular integration as described in Chapter 2 of Pozrikidis [18], and where $\boldsymbol{x}\left(r_{0}, z_{0}\right)$ is a fixed point located right on the boundary in the zeroth wave-section of the capillary, $\boldsymbol{y}^{\prime}\left(z^{\prime}, r^{\prime}\right)=k \hat{z}+\boldsymbol{y}$ is the variable point on boundary in the kth wave section of the capillary, $\boldsymbol{y}(z, r)$ is the variable point on the boundary in the zeroth wave section of the capillary, $d l$ is the differential arc length of the trace of the boundary and

$$
l(z)=\int_{0}^{z} \sqrt{\left[h^{\prime}(\epsilon)\right]^{2}+1} d \epsilon
$$

The contributions from the 4th, 5th and 6th terms in the right hand side of equation (5) are zero, since the velocity is zero on the tube surface and the function $Q(x, y)$ approaches zero at the end cross-sections for a long tube. Using equation (4) in equation (5), we can write the z-component velocity equation as follows:

$$
\begin{aligned}
u_{z}(\boldsymbol{x}) & =\int_{l(-1 / 2)}^{l(1 / 2)} d l(\boldsymbol{y}) \mathrm{M}_{z z}\left[z n_{z}(\boldsymbol{y})+f_{z}(\boldsymbol{y})\right]+\int_{l(-1 / 2)}^{l(1 / 2)} d l(\boldsymbol{y}) \mathrm{N}_{z z}(\boldsymbol{x}, \boldsymbol{y}) n_{z}(\boldsymbol{y})+\int_{l(-1 / 2)}^{l(1 / 2)} d l(\boldsymbol{y}) \mathrm{M}_{z r}(\boldsymbol{x}, \boldsymbol{y})\left[z n_{r}(\boldsymbol{y})+f_{r}(\boldsymbol{y})\right] \\
& +\int_{l(-1 / 2)}^{(l / 2)} d l(\boldsymbol{y}) \mathrm{N}_{z r}(\boldsymbol{x}, \boldsymbol{y}) n_{r}(\boldsymbol{y})+4 \pi h^{2}\left(\frac{1}{2}\right)
\end{aligned}
$$


where the last term in the right hand side of equation (6) comes from the 2 nd and 3 rd terms in the right hand side of equation (5), and where

$$
\begin{array}{ll}
\left.\mathrm{M}_{z z}(\boldsymbol{x}, \boldsymbol{y})=M_{z z}(\boldsymbol{x}, \boldsymbol{y})+\sum_{k=1}\left[M_{z z}(\boldsymbol{x}, k \hat{\mathbf{z}}+\boldsymbol{y})+M_{z z}(\boldsymbol{x},-k \hat{\mathbf{z}}+\boldsymbol{y})\right)\right] & \mathrm{N}_{z z}(\boldsymbol{x}, \boldsymbol{y})=\sum_{k=1} k\left[M_{z z}(\boldsymbol{x}, k \hat{\mathbf{z}}+\boldsymbol{y})-M_{z z}(\boldsymbol{x},-k \hat{\mathbf{z}}+\boldsymbol{y})\right] \\
\mathrm{M}_{z r}(\boldsymbol{x}, \boldsymbol{y})=M_{z r}(\boldsymbol{x}, \boldsymbol{y})+\sum_{k=1}\left[M_{z r}(\boldsymbol{x}, k \hat{\mathbf{z}}+\boldsymbol{y})+M_{z r}(\boldsymbol{x},-k \hat{\mathbf{z}}+\boldsymbol{y})\right] & \mathrm{N}_{z r}(\boldsymbol{x}, \boldsymbol{y})=\sum_{k=1} k\left[M_{z r}(\boldsymbol{x}, k \hat{\mathbf{z}}+\boldsymbol{y})-M_{z r}(\boldsymbol{x},-k \hat{\mathbf{z}}+\boldsymbol{y})\right]
\end{array}
$$

where the $M_{i j}$ are defined in Chapter 2 of Pozrikidis [18]. Since $M \sim \frac{1}{k}$ for large k [18], care must be taken in interpreting the sums in equation (7). By considering a force balance over one wave section of the tube and determining the behavior of the integrals in (6) at large $\mathrm{k}$, we find that for large $\mathrm{k}$

$$
\sum_{k=1}^{N}\left\{\frac{8 \pi}{k} \int_{l(-1 / 2)}^{l(1 / 2)} d l(y)\left[z n_{z}(z)+f_{z}(y)\right] h(z)-\frac{8 \pi}{k} \int_{l(-1 / 2)}^{l(1 / 2)} d l(y)\left(z-z_{0}\right) h(z) n_{z}(z)+\frac{4 \pi}{k} \int_{l(-1 / 2)}^{l(1 / 2)} d l(y) h^{2}(z) n_{r}(z)\right\}=0
$$

Subtracting Eq. (8) from Eq. (6), we obtain

$$
\begin{aligned}
u_{z}(\boldsymbol{x}) & =\frac{1}{4 \pi}\left[\int_{l(-1 / 2)}^{l(1 / 2)} d l(\boldsymbol{y}) \mathrm{M}_{z z}^{\prime}\left[z n_{z}(\boldsymbol{y})+f_{z}(\boldsymbol{y})\right]+\int_{l(-1 / 2)}^{l(1 / 2)} d l(\boldsymbol{y}) \mathrm{N}_{z z}^{\prime}(\boldsymbol{x}, \boldsymbol{y}) n_{z}(\boldsymbol{y})\right. \\
& \left.+\int_{l(-1 / 2)}^{l(1 / 2)} d l(\boldsymbol{y}) \mathrm{M}_{z r}(\boldsymbol{x}, \boldsymbol{y})\left[z n_{r}(\boldsymbol{y})+f_{r}(\boldsymbol{y})\right]+\int_{l(-1 / 2)}^{(l / 2)} d l(\boldsymbol{y}) \mathrm{N}_{z r}^{\prime}(\boldsymbol{x}, \boldsymbol{y}) n_{r}(\boldsymbol{y})+4 \pi h^{2}\left(\frac{1}{2}\right)\right]
\end{aligned}
$$

where $M_{z z}^{\prime}(x, y), N_{z z}^{\prime}(\boldsymbol{x}, \boldsymbol{y})$ and $N_{z r}^{\prime}(\boldsymbol{x}, \boldsymbol{y})$ are defined below and now converge like $\frac{1}{k^{3}}$ and

$$
\begin{gathered}
\left.\mathrm{M}_{z z}^{\prime}(\boldsymbol{x}, \boldsymbol{y})=M_{z z}(\boldsymbol{x}, \boldsymbol{y})+\sum_{k=1}\left[M_{z z}(\boldsymbol{x}, k \text { y })+M_{z z}(\boldsymbol{x},-k \mathbf{z}+\boldsymbol{y})\right)-\frac{8 \pi h(z)}{|k|}\right], \\
\mathrm{N}_{z z}^{\prime}(\boldsymbol{x}, \boldsymbol{y})=\sum_{k=1} k\left[M_{z z}(\boldsymbol{x}, k+\boldsymbol{y})-M_{z z}(\boldsymbol{x},-k \mathbf{z}+\boldsymbol{y})+\frac{8 \pi\left(z-z_{0}\right) h(z)}{k^{2}}\right] \\
\mathrm{N}_{z r}^{\prime}(\boldsymbol{x}, \boldsymbol{y})=\sum_{k=1} k\left[M_{z r}(\boldsymbol{x}, k+\boldsymbol{y})-M_{z r}(\boldsymbol{x},-k \mathbf{z}+\boldsymbol{y})-\frac{4 \pi h^{2}(z)}{k^{2}}\right]
\end{gathered}
$$

Setting the fluid velocity on the tube surface to zero results in equations

$$
\int_{l(-1 / 2)}^{l(1 / 2)} d l(\boldsymbol{y}) \mathrm{M}_{z z}^{\prime}(\boldsymbol{x}, \boldsymbol{y}) f_{z}(\boldsymbol{y})+\int_{l(-1 / 2)}^{l(1 / 2)} d l(\boldsymbol{y}) \mathrm{M}_{z r}(\boldsymbol{x}, \boldsymbol{y}) f_{r}(\boldsymbol{y})+\left\{I(x)+4 \pi h^{2}\left(\frac{1}{2}\right)\right\}=0
$$

where

$$
I(x)=\int_{l(-1 / 2)}^{l(1 / 2)} d l(\boldsymbol{y}) \mathrm{M}_{z z}^{\prime}(\boldsymbol{x}, \boldsymbol{y}) z n_{z}(\boldsymbol{y})+\int_{l(-1 / 2)}^{l(1 / 2)} d l(\boldsymbol{y}) \mathbb{N}_{z z}^{\prime}(\boldsymbol{x}, \boldsymbol{y}) n_{z}(\boldsymbol{y})+\int_{l(-1 / 2)}^{l(1 / 2)} d l(\boldsymbol{y}) \mathrm{M}_{z r}(\boldsymbol{x}, \boldsymbol{y}) z n_{r}(\boldsymbol{y})+\int_{l(-1 / 2)}^{l(1 / 2)} d l(\boldsymbol{y}) \mathbb{N}_{z r}^{\prime}(\boldsymbol{x}, \boldsymbol{y}) n_{r}(\boldsymbol{y})
$$

A similar treatment of the $r$-component of equation (1) results in equation (10).

The $r$-component of equation (1) is

$$
\int_{l(-1 / 2)}^{l(1 / 2)} d l(\boldsymbol{y}) \mathbb{M}_{r z}(\boldsymbol{x}, \boldsymbol{y}) f_{z}(\boldsymbol{y})+\int_{l(-1 / 2)}^{l(1 / 2)} d l(\boldsymbol{y}) \mathbb{M}_{r r}(\boldsymbol{x}, \boldsymbol{y}) f_{r}(\boldsymbol{y})+J(\boldsymbol{x})=0
$$


where,

$$
J(x)=\int_{l(-1 / 2)}^{l(1 / 2)} d l(\boldsymbol{y}) \mathrm{M}_{r z}(\boldsymbol{x}, \boldsymbol{y}) z n_{z}(\boldsymbol{y})+\int_{l(-1 / 2)}^{l(1 / 2)} d l(\boldsymbol{y}) \mathbb{N}_{r z}^{\prime}(\boldsymbol{x}, \boldsymbol{y}) n_{z}(\boldsymbol{y})+\int_{l(-1 / 2)}^{l(1 / 2)} d l(\boldsymbol{y}) \mathrm{M}_{r r}(\boldsymbol{x}, \boldsymbol{y}) z n_{r}(\boldsymbol{y})+\int_{l(-1 / 2)}^{l(1 / 2)} d l(\boldsymbol{y}) \mathbb{N}_{r r}(\boldsymbol{x}, \boldsymbol{y}) n_{r}(\boldsymbol{y})
$$

and

$$
\begin{gathered}
\mathrm{M}_{r z}(\boldsymbol{x}, \boldsymbol{y})=M_{r z}(\boldsymbol{x}, \boldsymbol{y})+\sum_{k=1}\left[M_{r z}(\boldsymbol{x}, k \hat{\mathbf{z}}+\boldsymbol{y})+M_{r z}(\boldsymbol{x},-k \hat{\mathbf{z}}+\boldsymbol{y})\right] \\
\mathrm{N}_{r z}^{\prime}(\boldsymbol{x}, \boldsymbol{y})=\sum_{k=1} k\left[M_{r z}(\boldsymbol{x}, k \hat{\mathbf{z}}+\boldsymbol{y})-M_{r z}(\boldsymbol{x},-k \hat{\mathbf{z}}+\boldsymbol{y})+\frac{4 \pi h(z) h\left(z_{0}\right)}{k^{2}}\right] \\
\mathrm{M}_{r r}(\boldsymbol{x}, \boldsymbol{y})=M_{r r}(\boldsymbol{x}, \boldsymbol{y})+\sum_{k=1}\left[M_{r r}(\boldsymbol{x}, k \hat{\mathbf{z}}+\boldsymbol{y})+M_{r r}(\boldsymbol{x},-k \hat{\mathbf{z}}+\boldsymbol{y})\right] \\
\mathrm{N}_{r r}(\boldsymbol{x}, \boldsymbol{y})=\sum_{k=1} k\left[M_{r r}(\boldsymbol{x}, k \hat{\mathbf{z}}+\boldsymbol{y})-M_{r r}(\boldsymbol{x},-k \hat{\mathbf{z}}+\boldsymbol{y})\right]
\end{gathered}
$$

In order to solve equations (9) and (10) for obtaining components of surface force, the surface of the tube is discretized along the $z$-axis into $\mathrm{N}$ elements and the line integrals are approximated using the trapezoidal rule. Although the functions $M_{i j}(x, y)$ are singular when $y=x$, the singularities are integrable in the sense of the Cauchy principal value. Therefore, the integrals are evaluated by subtracting the immediate neighborhood of the singular point from the integration domain, integrating the kernel analytically in the region, and then adding the result to the integral computed numerically over the remainder of the domain. For pressure drop $\Delta p$ across one wave section, integral equations (9) and (10) represent a linear system of $2 \mathrm{~N}$ algebraic equations for the $2 \mathrm{~N}$ unknown components of the force distribution on the tube surface, $f=\left(f_{z}, f_{r}\right)$.

These equations are solved using an International Mathematics and Statistics Library (IMSL) routine. As described above, once the force on the tube surface is known, it can be used to calculate tangential force on the tube wall and the velocity distribution inside the tube domain.

\section{Results and Discussion}

In this section, the detailed velocity and tangential force distribution for a wide range of dimensionless amplitude and wave-length of corrugation are enumerated. The flow structure within the capillary was analyzed elaborately.

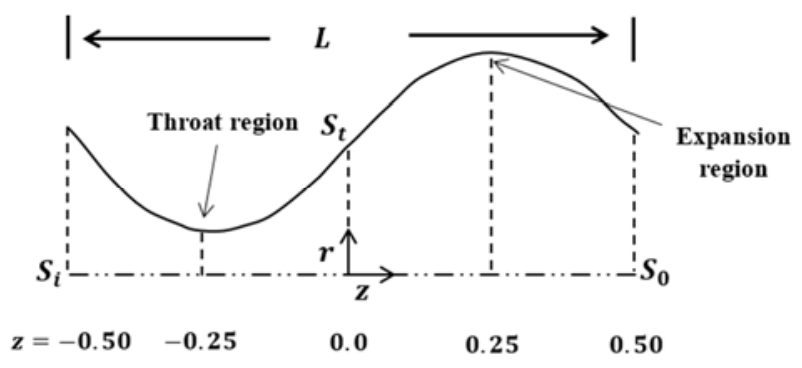

Figure 2. Profile of the sinusoidally constricted capillary.
Figure 2 represents the profile of a sinusoidally constricted periodic tube in the upper region only, since the tube is axisymmetric. We have analyzed our detailed velocity distribution in throat region and expansion region within this periodic tube.

At first, the effect of corrugation amplitude on the velocity field is considered. Figures 3, 4 and 5 show the axial velocity profiles at different axial positions for distinct corrugation amplitudes, $A=0.1 A=0.3, A=0.6$ respectively and for a fixed wavelength $L=1.0$. From Figure 3 , we can easily identify that the axial velocity profile is nearly parabolic and the axial velocity profiles at different cross-sectional areas are indistinguishable for a fixed wavelength. As the profile amplitudes slightly increases ( $A=0.3, A=0.6$ ), the axial velocity profiles in various cross-sectional areas are slightly distinguishable which can be seen in Figures 4 and 5 .

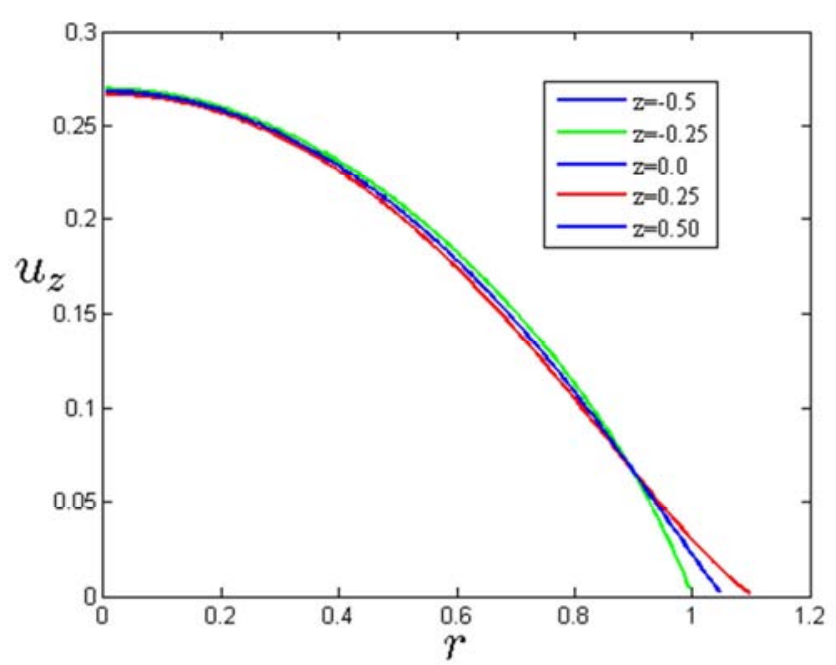

Figure 3. Axial velocity profiles at different axial positions for $A=0.1$ and $L=1.0$. 


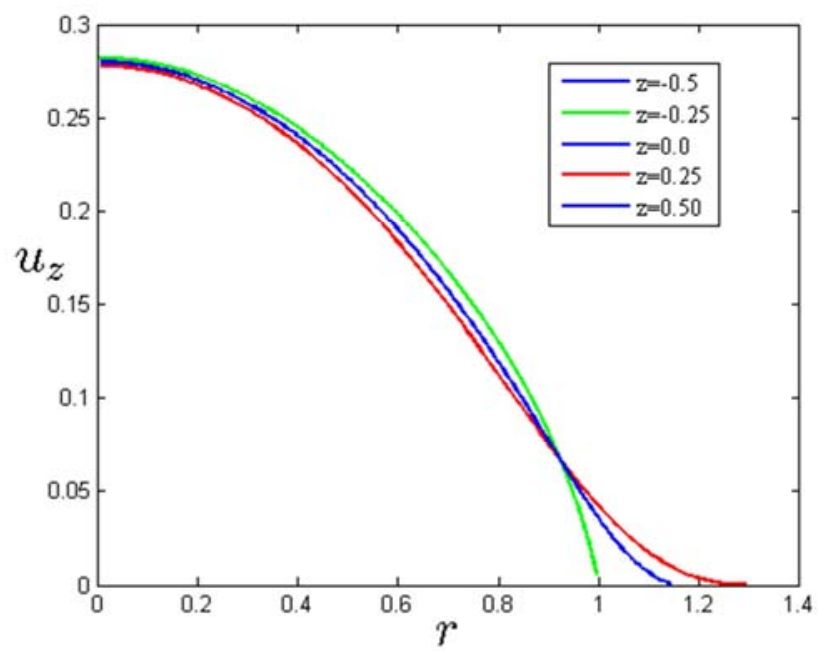

Figure 4. Axial velocity profiles at different axial positions for $A=0.3$ and $L=1.0$.

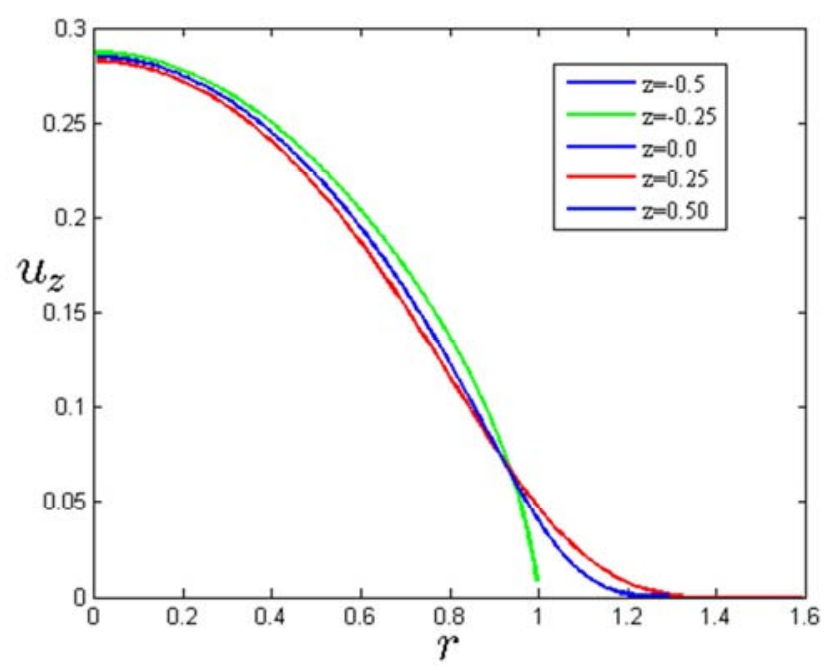

Figure 5. Axial velocity profiles at different axial positions for $A=0.6$ and $L=1.0$.

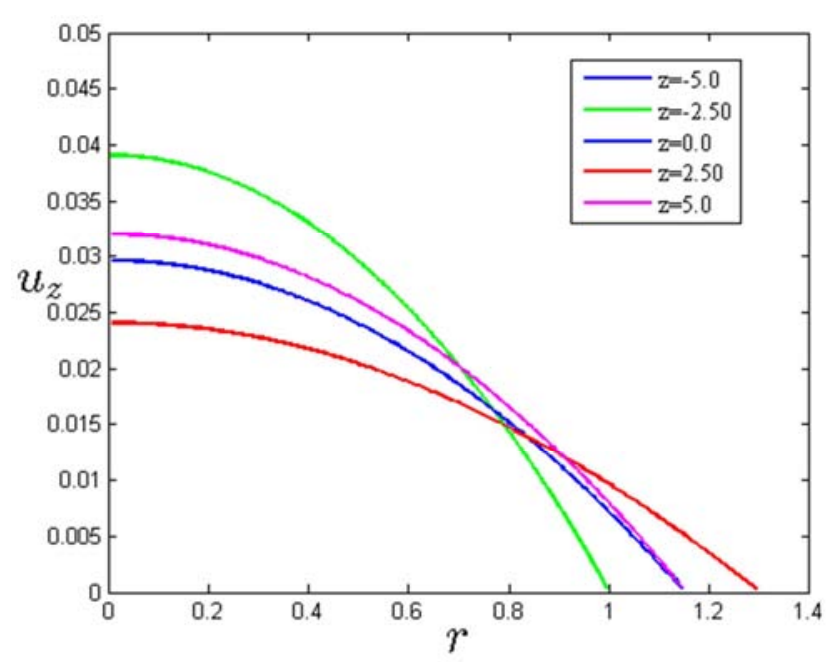

Figure 6. Axial velocity profiles at different axial positions for $A=0.3$ and $L=5.0$.

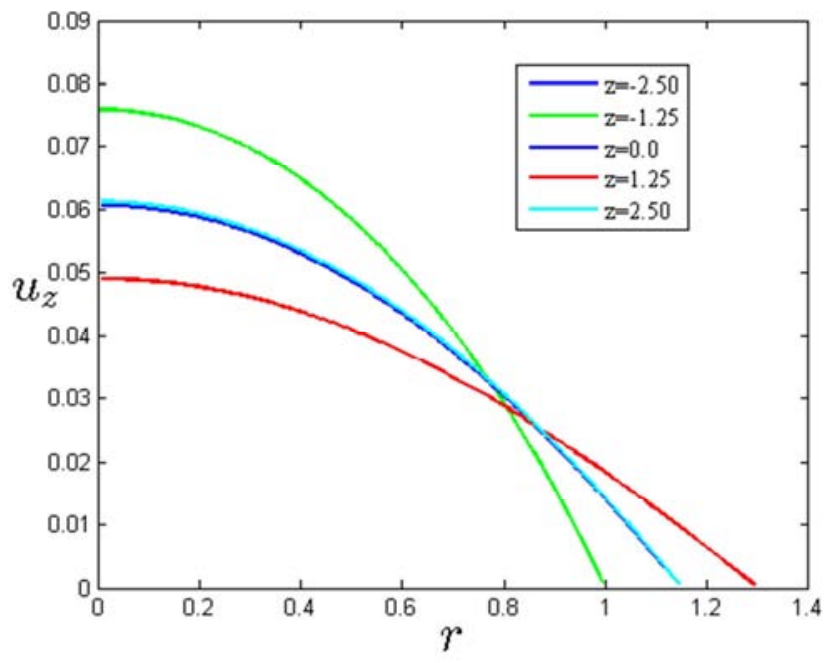

Figure 7. Axial velocity profiles at different axial positions for $A=0.3$ and $L=10.0$.

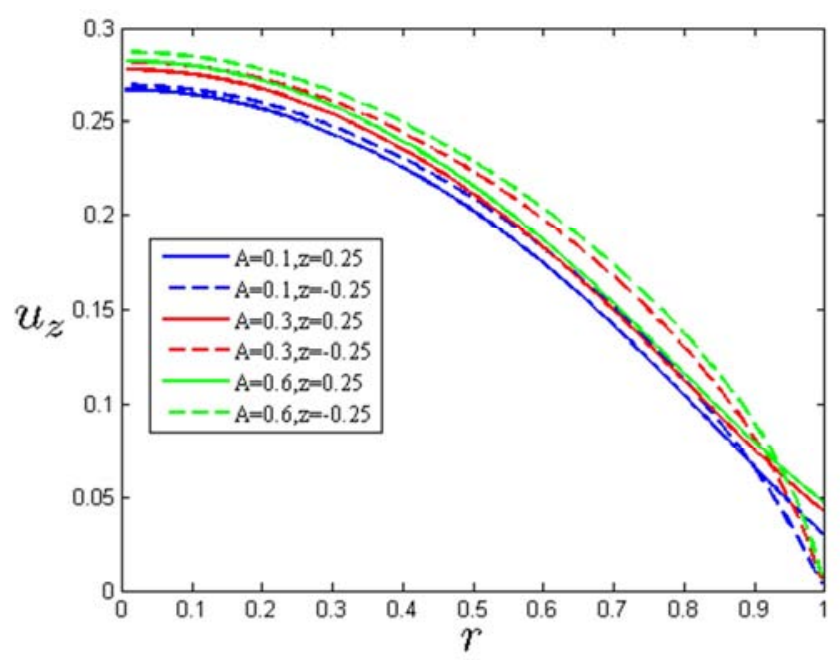

Figure 8. Effect of corrugation amplitude on the axial component of velocity at $z=0.25$ and $z=-0.25$ for $L=1.0$.

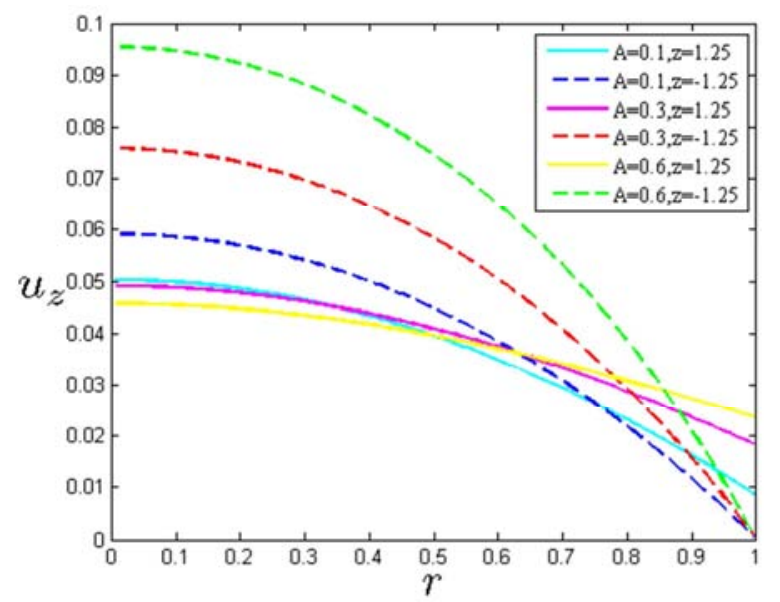

Figure 9. Effect of corrugation amplitude on the axial component of velocity at $z=-1.25$ and $z=1.25$ for $L=5.0$.

Figures 4, 6 and 7 show the axial velocity profiles at different axial positions for different wavelengths $L=1.0$, 
$L=5.0$ and $L=10.0$ respectively with fixed amplitude $A=0.3$. Needless to say that the axial velocity profile exhibit almost similar behavior at different cross-sections for fixed wavelength $L=1.0$ (Figures 3, 4, 5). But after increasing the wavelength it is quite fare to declare that axial velocity profile at different axial positions started to decrease with increasing wavelength and each velocity curves are more distinguishable than each other's.

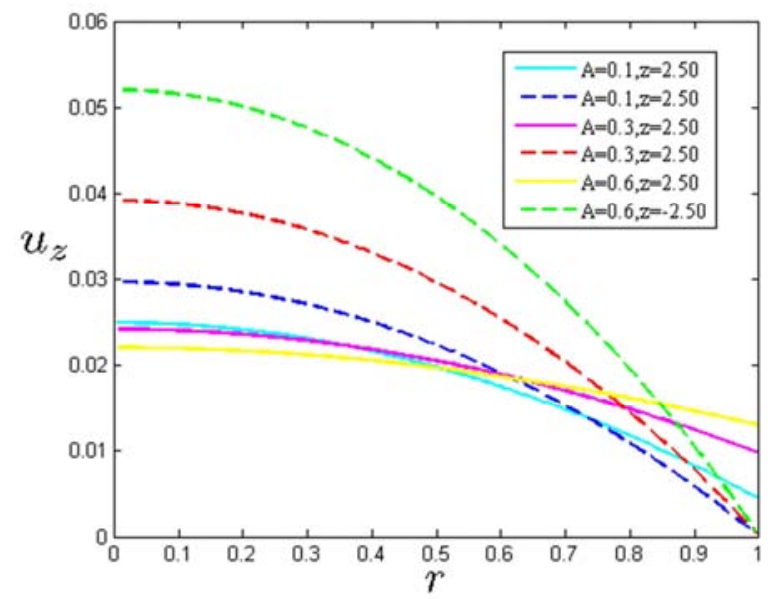

Figure 10. Effect of corrugation amplitude on the axial component of velocity at $z=2.50$ and $z=-2.50$ for $L=10.0$.

Now, we have considered the effect of corrugation amplitude on the axial component at the throat $(z=-0.25)$ and expansion $(z=0.25)$ regions for wavelength of corrugation $L=1.0$, which is shown in Figure 8 . The solid lines are for the expansion region while the dashed line are for the throat region. It can be easily identified that the axial velocity profile in the throat region is started to increase where as the axial velocity profile in the expansion region is started to decrease, after increasing corrugation amplitude.

Figures 9 and 10 represent the axial velocity profile at throat and expansion regions for wavelengths $L=5.0$ and $L=10.0$ respectively. The solid lines are for the expansion region while the dashed line are for the throat region. Here, we can observe that after increasing the wavelength, the axial velocity curves at both throat and expansion region for different amplitudes can be differentiated from each other than the previous one's (see Figure 3) and the effect of the curves are more distinguishable. Also, we can find that axial velocity is started to decrease when the wavelength is increased.

Figure 11 shows the radial component of velocity across the converging $(z=0.5)$ and diverging $(z=0.0)$ cross sections of the capillary for a range of amplitudes. The solid lines are for the diverging region while the dashed line are for the converging region. This figure shows that when the corrugation amplitudes is increased, maximum radial velocity started to plod away from the tube wall for wavelength $L=1.0$. But when we increase the wavelength from $L=1.0$. to $L=5.0$, radial velocity at converging region is started to increased but it is decreased in the diverging region. This phenomenon is represented in Figure 12. Now, we can easily conclude that the maximum radial velocity occurs at diverging cross- section and the minimum radial velocity occurs at converging cross-section. We can also found that if wavelength of corrugation is increased, the radial velocity in both regions started to decrease from their previous quantities.

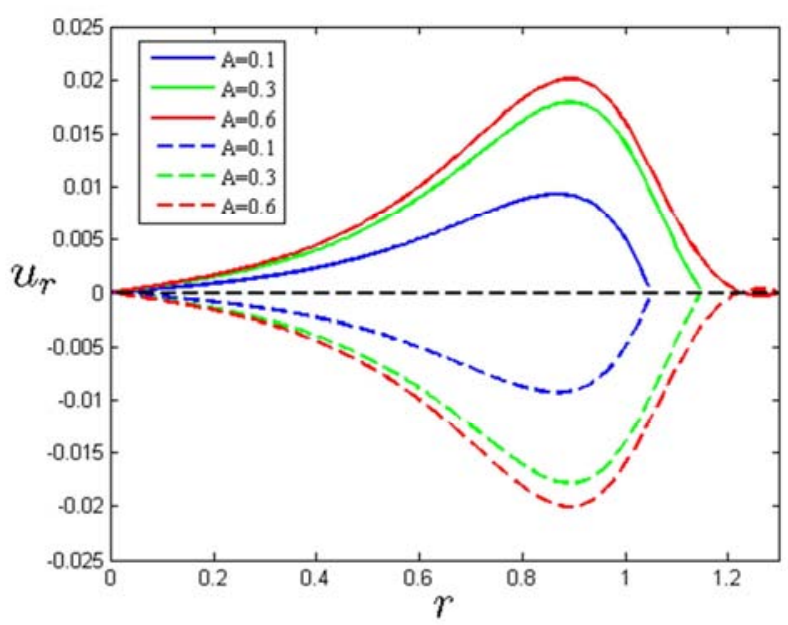

Figure 11. Effect of corrugation amplitude on the radial velocity at $z=0.0$ and $z=0.50$ for $L=1.0$

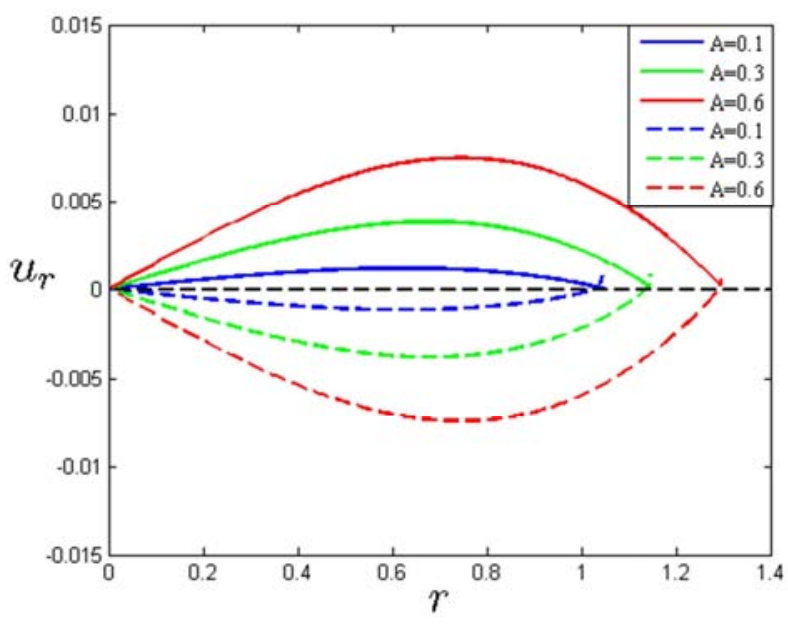

Figure 12. Effect of corrugation amplitude on the radial velocity at $z=0.0$ and $z=2.50$ for $L=5.0$

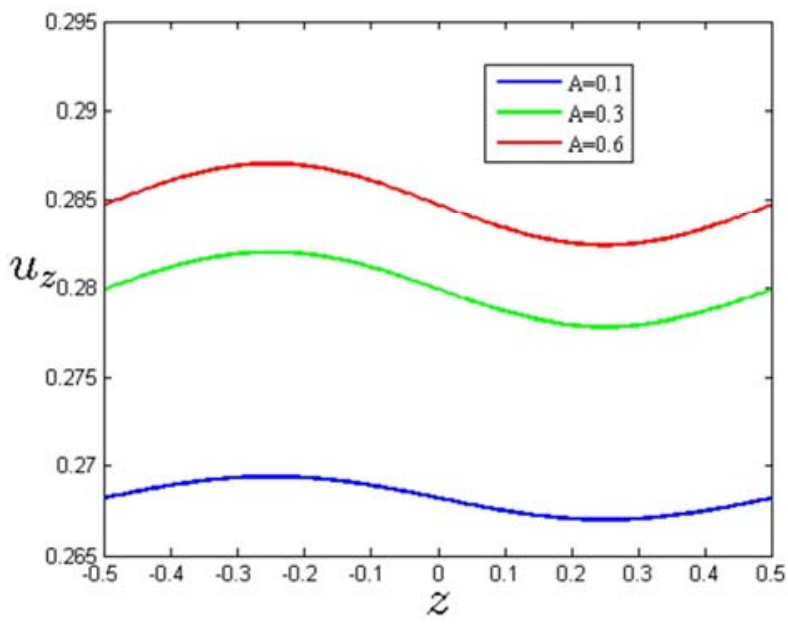

Figure 13. Axial variation of the centreline velocity for different amplitudes with fixed wavelength for $L=1.0$. 


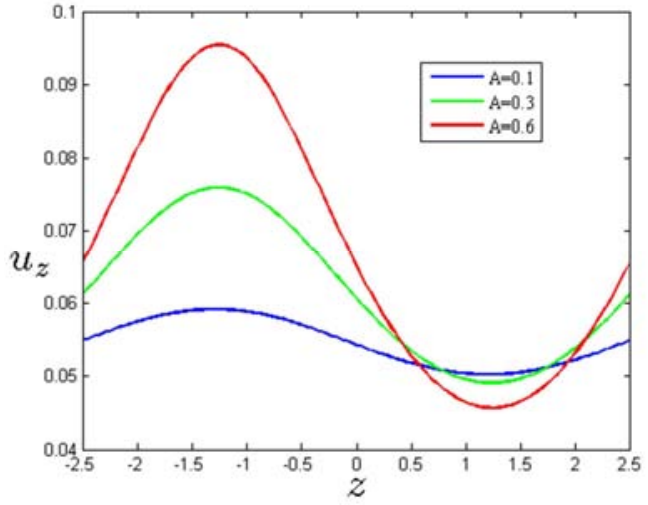

Figure 14. Axial variation of the centreline velocity for different amplitudes with fixed wavelength for $L=5.0$.

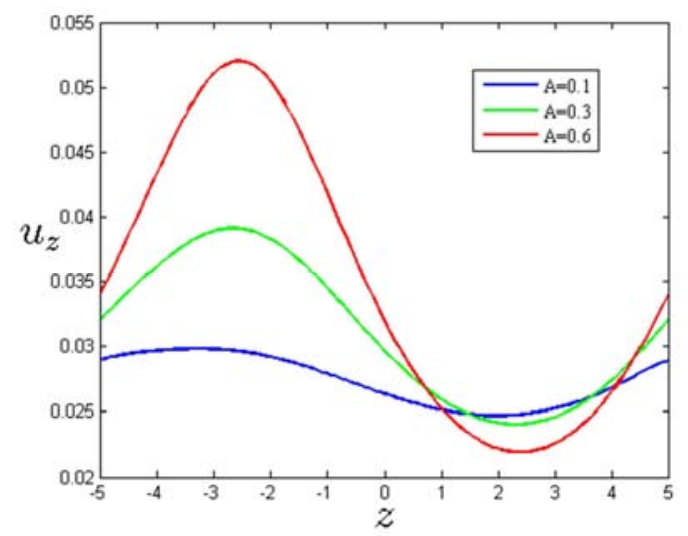

Figure 15. Axial variation of the centreline velocity for different amplitudes with fixed wavelength for $L=10.0$.

Axial variation of the central line velocity for different amplitudes with fixed wavelength $L=1.0$ is shown in Figure 13. This figure shows that the largest axial velocity within the capillary occurs at the throat for all corrugation amplitudes. When we increase the wavelength, it can be easily observed that the axial variation of central line velocity for three different amplitudes are more deviated (Figures 14 and 15). Also, centerline velocity is started to decrease when we increase the wavelength of corrugation. These effects can be seen in Figureas 14 and 15.

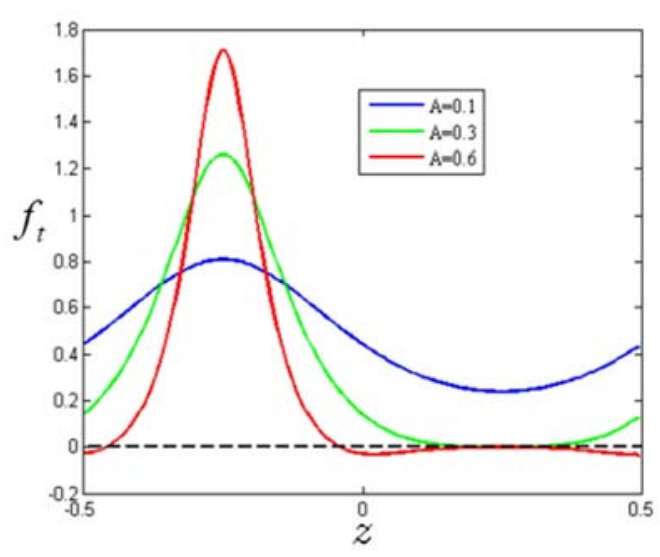

Figure 16. Effect of corrugation amplitude on the wall tangential force distribution with for $L=1.0$.

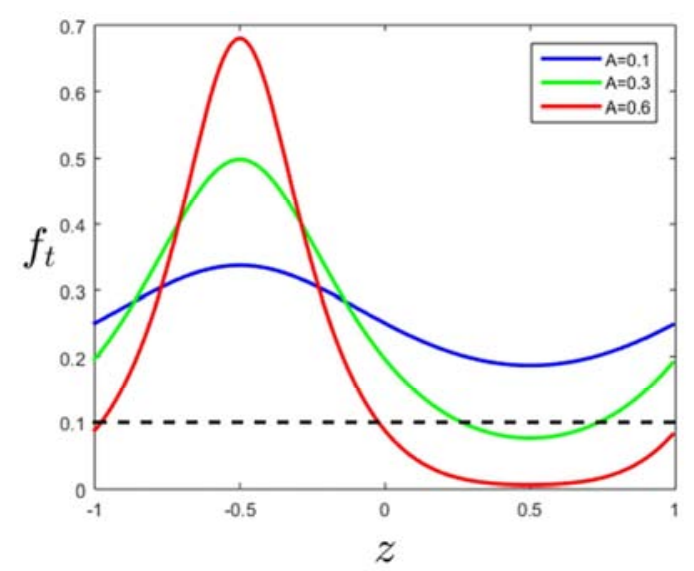

Figure 17. Effect of corrugation amplitude on the wall tangential force distribution with for $L=5.0$.

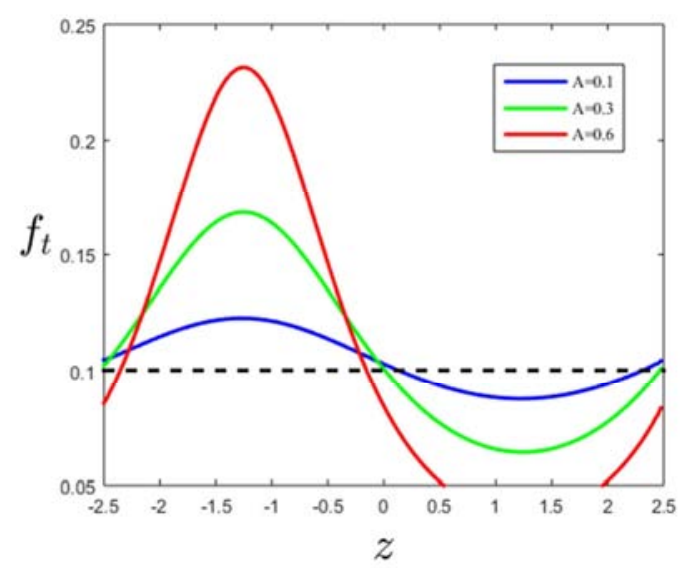

Figure 18. Effect of corrugation amplitude on the wall tangential force distribution with for $L=10.0$.

The tangential force distribution on the capillary wall is presented in Figure 16 for various amplitudes with fixed wavelength $L=1.0$. This figure shows that when we increased the amplitudes of corrugation, the tangential force at the throat region is onerously increased and the tangential force at the expansion region is more likely to be comparatively impercipient with respect to the capillary geometry. Figures 17 and 18 represent the tangential force distribution on the capillary wall for different wavelengths $L=5.0$ and $L=10.0$. These two figures indicate that as the wavelength of corrugation is increased, the high tangential force at the throat region started to reduce from its previous position and it becomes more generalized around the throat region. The wall tangential force in the expansion region is more likely to perceive an expansion comparated to capillary geometry with increasing amplitude. It can be observed that the tangential force $f_{t}$ is greater in the constricted region than in the expansion region. We can also observed from Figures 16-18 that the tangential force increases with increasing amplitude and decreases with decreasing wavelength.

In Figure 19, we have compared our calculated tangential force distribution on the tube surface for the two distinct expansion amplitudes $A=0.3$ and $A=0.6$, with the results 
produced by Hemmat and Borhan [3] for the case of their results for each tube profile. sinusoidal profile. We have found excellent agreement with

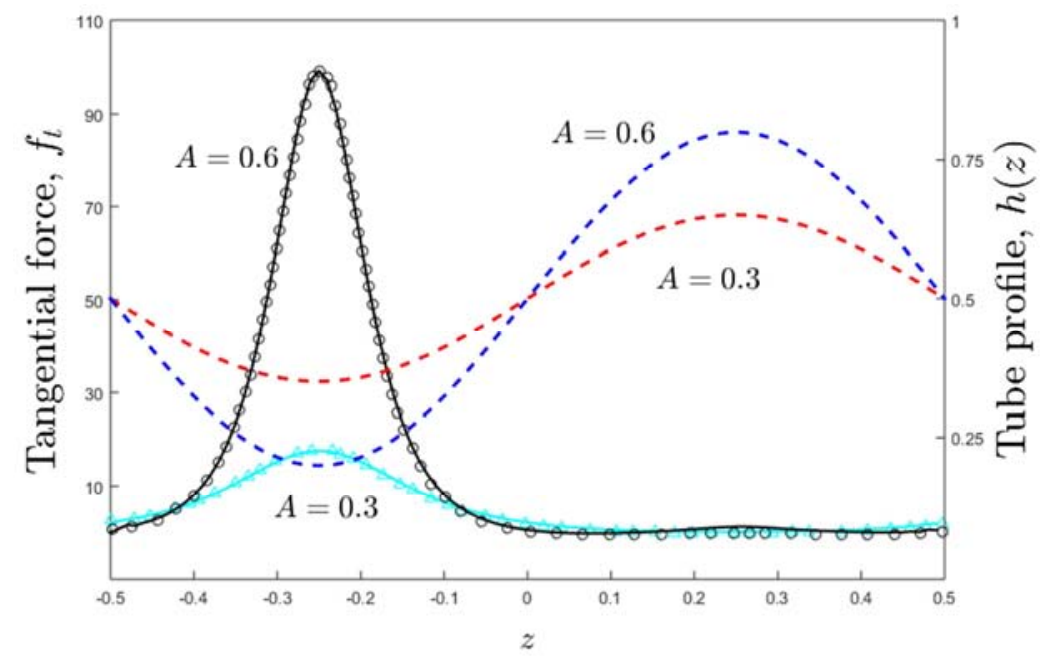

Figure 19. Comparison between our tangential force calculations [17] and that of Hemmat and Borhan [3] for a sinusoidal tube shape with $A=0.3$ and $A=0.6$. The dashed line shows the two tube profiles (right hand axis). The solid line show our calculations for the amplitudes $A=0.3$ and $A=0.6$, and the circle ( $A=0.6)$ and triangle ( $A=0.3)$ show the calculation of Hemmat and Borhan [3] (left-hand axis).

\section{Conclusions}

In this paper, we have investigated the creeping flow of viscous incompressible fluid through sinusoidally periodic tube by using boundary element method. We have analyzed the different velocity distribution for different wavelengths and corrugation amplitudes. We have enumerated the axial velocity profile for the variation of corrugation amplitude and found that the highest axial velocity is at throat region and lowest axial velocity is at expansion region. Also, we have analyzed the axial velocity profile at different axial positions for different wavelength and corrugation amplitudes. Similarly, we have examined the radial velocity profile for different corrugation amplitudes and found that the maximum radial velocity occurs at diverging cross- section and minimum radial velocity occurs at converging crosssection. We have also calculated the tangential force distribution for different corrugation amplitudes. The tangential force distribution exhibit differences for each amplitude. We observed that the tangential force is greater in the constricted region than in the expansion region. In addition, axial velocity, radial velocity, centreline velocity and tangential force increase with increasing amplitude and decrease with increasing wavelength. Finally, we have compared our results with the result of Hemmat and Borhan [3] and found excellent agreement with them.

\section{References}

[1] Chow, J. C. F. and Soda, K. (1972) Laminar flow in tubes with constriction. Physics of Fluids. 15: 1700-1706.

[2] Deiber, J. A. and Schowalter, W. R. (1979) Flow through tubes with sinusoidal axial variations in diameter. International Journal of American Institute of Chemical
Engineers. 25: 638-645.

[3] Hemmat, M. and Borhan, A. (1995) Creeping flow through sinusoidally constricted capillaries. Physics of Fluids. 7: 21112121.

[4] Ralph, M. E. (1987) Steady flow structures and pressure drops in wavy-walled tubes. Journal of Fluids Engineering. 109: 255-261.

[5] Sisavath, S., Jing, X. and Zimmerman, R. W. (2001) Creeping flow through a pipe of varying radius. Physics of Fluids. 13: 2762-2772.

[6] Tilton, J. N. and Payatakes, A. C. (1984) Collocation solution of creeping Newtonian flow through sinusoidal tubes: a correction. International Journal of American Institute of Chemical Engineers 30: 1016-1021.

[7] Islam, N. (2016) Fluid flow and particle transport through periodic capillaries. PhD dissertation, University of South Australia, Australia.

[8] Sisavath, S., Jing, X. and Zimmerman, R. W. (2001) Creeping flow through a pipe of varying radius. Physics of Fluids. 13, 2762-2772.

[9] Hagen, G. H. L. (1839) Uber die Bewegung des Wassers in engen cylindrischen Rohren. Poggendorf's Annalen der Physik und Chemie. 46: 423-42.

[10] Poiseuille, J. L. M. (1830) Recherches sur les causes du mouvement du sang dans les veincs. Journal of Clinical and Experimental Pathology 10: 277-295.

[11] Rothstein, J. P. and McKinley, G. H. (2001) The axisymmetric contraction expansion: the role of extensional rheology on vortex growth dynamics and the enhanced pressure drop. Journal of Non-Newtonian Fluid Mechanics 98: 33-63.

[12] Subramanya, K. (1982) Flow in Open Channels, $3^{\text {rd }}$ ed.; Tata McGraw-Hill Education, New Delhi, India. 
[13] Moffatt, H. K. (1964) Viscous and resistive eddies near a sharp corner. Journal of Fluid Mechanics. 18: 1-18.

[14] Forrester, J. H. and Young, D. F. (1970) Flow through a converging-diverging tube and its implications in occlusive vascular disease. Journal of Biomechanics 3: 297-305.

[15] Leneweit, G. and Auerbach, D. (1999) Detachment phenomena in low Reynolds number flows through sinusoidally constricted tubes. Journal of Fluid Mechanics 387: $129-150$

[16] Nishimura, T., Bian, Y., Matsumoto, Y. and Kunitsugu, K.
(2003) Fluid flow and mass transfer characteristics in a sinusoidal wavy-walled tube at moderate Reynolds numbers for steady flow. International Journal of Heat and Mass Transfer. 39 (3): 239-248.

[17] Islam, N., Bradshaw-Hajek, B. H., Miklavcic, S. J. and White, L. R. (2015) The onset of recirculation flow in periodic capillaries: Geometric effects. European Journal of Mechanics B/Fluids. 53: 119-128.

[18] Pozrikidis, C. (1992) Boundary Integral and singularity Methods for Linearised Viscous Flow, Cambridge University Press, Cambridge, U.K. 